\title{
MICROWAVE INDICATOR OF POTENTIAL GEOEFFECTIVENESS AND MAGNETIC FLUX-ROPE STRUCTURE OF A SOLAR ACTIVE REGION
}

\author{
A.V. Kudriavtseva \\ Institute of Solar-Terrestrial Physics SB RAS, \\ Irkutsk, Russia, kudryavtseva@isz..irk.ru

\section{I.I. Myshyakov} \\ Institute of Solar-Terrestrial Physics SB RAS, \\ Irkutsk, Russia,ivan_m@isz.irk.ru
}

\author{
A.M. Uralov \\ Institute of Solar-Terrestrial Physics SB RAS, \\ Irkutsk,Russia,uralov@iszf.irk.ru \\ V.V. Grechnev \\ Institute of Solar-Terrestrial Physics SB RAS, \\ Irkutsk, Russia,grechnev@isz.irk.ru
}

\begin{abstract}
We analyze the presence of a microwave neutral-line-associated source (NLS) in a super-active region NOAA 12673, which produced a number of geoeffective events in September 2017. To estimate the NLS position, we use data from the Siberian Radioheliograph in a range $4-8 \mathrm{GHz}$ and from the Nobeyama Radioheliograph at $17 \mathrm{GHz}$. Calculation of the coronal magnetic field in a non-linear force-free approximation has revealed an extended structure consisting of interconnected magnetic flux ropes, located practically along the entire length of the main polarity inversion line of
\end{abstract}

the photospheric magnetic field. NLS is projected into the region of the strongest horizontal magnetic field, where the main energy of this structure is concentrated. During each X-class flare, the active region lost magnetic helicity and became a CME source.

Keywords: solar active regions, magnetic fields, microwave emission, solar flare forecast.

\section{INTRODUCTION}

One of the objectives of the under-construction Siberian Radioheliograph (SRH) [Lesovoi et al., 2017; Altyntsev et al., 2020] is to assess the ability of solar active regions (ARs) to produce energetic flares and coronal mass ejections (CMEs). The final configuration of SRH will be able to map ARs with an angular resolution from 30 " to $7 "$ in a frequency range from 3 to 24 $\mathrm{GHz}$. A candidate for a microwave indicator of AR potential geoeffectiveness in this frequency range is a microwave neutral-line-associated source (NLS).

In the atmosphere of solar ARs there are long-lived microwave sources of two types. The first type includes stationary sources in magnetic fields over sufficiently large unipolar sunspots, sunspot-associated sources. Their polarized emission is dominated by thermal gyroresonance mechanism. The emissivity and optical thickness of gyroresonance levels corresponding to the observed microwave emission increase rapidly with increasing plasma temperature. As such, the emission of a sunspot-associated source is concentrated in the hot corona above the cold chromosphere and has a flux density spectrum steeply decreasing with increasing frequency. The larger the sunspot and its magnetic flux, the higher the radio frequency at which the sunspotassociated source may be detected.

Quasi-stationary sources of the second type are located above the polarity inversion line of the photospheric magnetic field and can be conventionally classified into two groups. The first group comprises diffuse weakly polarized sources with low brightness. Such sources always appear when bipolar magnetic structures emerge into the solar corona. Their optically thin emission is determined by Coulomb collisions of particles in hot plasma, confined in magnetic loops rooted to the photosphere. The second group includes compact sources over the neutral line of the magnetic field, first described in [Quiñones et al., 1975; Kundu et al., 1977]. Their brightness temperatures are often higher than those of usual gyroresonance sources over sunspots.

NLSs were occasionally recorded during solar observations by large multipurpose radio telescopes: Westerbork Synthesis Radio Telescope (WSRT), Very Large Array (VLA), RATAN-600, and were also investigated using regular observations made with the Siberian Solar Radio Telescope (SSRT) [Smolkov et al., 1986; Grechnev et al., 2003] and the Nobeyama Radioheliograph (NoRH) [Nakajima et al., 1994]. WSRT observed NLSs at $5 \mathrm{GHz}$; VLA, at 4.9, 8.4, and $15 \mathrm{GHz}$; SSRT, at $5.7 \mathrm{GHz}$; RATAN-600, at several frequencies; NoRH, at $17 \mathrm{GHz}$ and in one case even at $34 \mathrm{GHz}$. Results of these studies have been discussed in several papers [Kundu, Velusamy, 1980; Kundu et al., 1981; Kundu, Alissandrakis, 1984; Strong et al., 1984; Gelfreikh, 1985; Akhmedov et al., 1986; Chiuderi Drago et al., 1987; Borovik et al., 1989; Vatrushin, Korzhavin, 1989; Sych et al., 1993; Uralov et al., 1996, 1998, 2000, 2006a, 2006b, 2007, 2008; Lee et al., 1997; Rudenko et al., 2007; Bogod et al., 2012; Yasnov, 2014; Abramov-Maximov et al., 2015; Kuznetsov et al., 2016; Bakunina et al., 2017; Zaitsev, 2019].

NLS is the main source of microwave fluctuations in the AR, where it resides, and appears, where oppositepolarity sunspots most closely approach each other. In photospheric magnetograms, this approaching may look like a collision of emerging bipolar magnetic fluxes either with one another or with already-existing ones. This process is accompanied by shear and rotational motions of photospheric plasma, which occupy eventually the entire AR. These factors determine the non-potential component and helicity of the AR magnetic field. If such colliding oppo- 
site-polarity magnetic fluxes make up a $\delta$-configuration (bipolar sunspots within common penumbra), NLS can be detected at high frequencies (e.g., at $17 \mathrm{GHz}$ ). Otherwise, it either does not manifest itself at all or appears at lower frequencies (e.g., below $10 \mathrm{GHz}$ ). Of practical importance is the experimental fact that there are bright NLSs at 17 $\mathrm{GHz}$ in the ARs that produce GOES X-class flares. If in this AR no NLS is seen at $17 \mathrm{GHz}$, but it is observed, for example, at $5.7 \mathrm{GHz}$, only M-class flares can occur. NLS appears before a powerful flare or a series of such flares with a lead time from a few hours to two days. The NLS at $17 \mathrm{GHz}$ recovers after a flare and exists as long as a series of X-class flares continues. Moreover, an $\mathrm{X}$-class flare at $17 \mathrm{GHz}$ may commence in the vicinity of NLS. These circumstances can be used as predictors of $\mathrm{X}$-class flares.

Being a consequence of approaching of two opposite-polarity sunspots, the NLS at $17 \mathrm{GHz}$ often resembles a typical sunspot-associated radio source and can adjoin it. The degree of circular polarization of such a complex source, unresolved in microwave images, can reach $90 \%$, with the polarization sign corresponding to a sunspot with the strongest magnetic field and magnetic flux. On the one hand, this similarity is consistent with the assumption that the gyroresonance thermal mechanism predominates in NLS emission - at least in these simple cases. On the other hand, this similarity complicates the identification of NLS at $17 \mathrm{GHz}$. Direct comparison of microwave images with observed line-ofsight photospheric magnetograms also does not always allow a reliable identification of NLS because of projection displacement of the magnetic inversion line inside AR.

The NLS identification method we use involves superposing microwave images and the distribution of coronal magnetic components calculated at a given height of the AR atmosphere. This approach is based on the fact that the NLS brightness center is projected onto the neutral line of the vertical magnetic component, where the horizontal component is maximum [Uralov et al., 2006a, 2006b].

This article has two goals:

1. To refine the method of identifying NLS, using vector magnetograms and observations of a solar geoeffective region at the first stage of the under-construction Siberian Radioheliograph. The SRH frequency range at this stage is $4-8 \mathrm{GHz}$, and the angular resolution is low, about 100". Therefore, as reference we use NoRH images obtained with a higher angular resolution at $17 \mathrm{GHz}$.

2. To estimate the NLS position at $17 \mathrm{GHz}$ relative to the AR flux-rope magnetic structure calculated in the nonlinear force-free approximation. Current-carrying coronal structures, i. e. magnetic flux ropes, are known to be drivers of eruptive flares and the main components of CMEs.

\section{ACTIVE REGION NOAA 12673}

The first stage of SRH started operating at the decay phase of solar cycle 24 . That is why we could observe only one AR 12673, which produced a series of X-class flares in September 2017. The X9.3 flare on September 6 was the strongest in solar cycle 24. Overall, on September 4-10 this super-active region produced four X-class flares, many
M-class flares, intense proton fluxes, a strong geomagnetic storm on September 7-9 with Dst=-124 nT, a considerable Forbush decrease, and a ground level enhancement of cosmic-ray intensity on September 10 . We present only reliable data as adjustment of the SRH hardware systems was not completed in that period. For this reason and because of insufficient angular resolution of the first stage of $\mathrm{SRH}$, we do not estimate the degree of polarization and the spectral slope of radio sources.

NOAA 12673 appeared on the east limb on August 28 as a single $\alpha$-spot, with the diameter of its umbra not exceeding $10^{\prime \prime}$. The magnetic field of up to $3500 \mathrm{G}$ expected in the corona above such a sunspot can produce a faint gyroresonance source at frequencies below $8 \mathrm{GHz}$ and cannot at $17 \mathrm{GHz}$. On September 3, new bipolar magnetic fluxes began to emerge at an intensive rate. Their expansion on September 4 led to the collision of their western edge with the existing $\alpha$-spot, to the flow around it, and to the formation of $\delta$-configuration. The $\alpha$-spot remained static. From September 4 on, the AR became a source of noticeable emission at $17 \mathrm{GHz}$. On September 5 and on the following days, the main component of quasi-stationary emission of NOAA 12673 at $17 \mathrm{GHz}$ was NLS, which is also confirmed by the analysis of one-dimensional microwave observations of NOAA 12673 by RATAN-600 those days [Borovik et al., 2018]. According to Verma [2018], "The enhanced flare activity has its origin in the head-on collision of newly emerging flux with an already existing regular, $\alpha$-spot". These circumstances caused considerable shear motions and long-term dynamic compression of magnetic fluxes inside the $\delta$-configuration. This resulted in an unusually high strength of the photospheric magnetic field up to 5570 G [Wang et al., 2018], almost entirely concentrated in the transverse component on the polarity inversion line.

The presence of large magnetic fluxes with such high strength in NOAA 12673 explains the appearance of the quasi-stationary compact source with a brightness temperature of $\sim 0.4 \mathrm{MK}$ at $34 \mathrm{GHz}$ in the vicinity of the neutral line of the photospheric magnetic field. As we know, this has not been observed before. Numerical simulation of the magnetic structure and gyroresonance emission of NOAA 12673 in [Anfinogentov et al., 2019] has shown that the NLS observed at 17 and $34 \mathrm{GHz}$ corresponded to the unusually high magnetic field strength detected at the photospheric level.

Abramov-Maximov et al. [2018] have suggested that there are magnetic flux ropes in the magnetosphere of NOAA 12673. Their real presence and the conditions necessary for their eruption have been discussed in [Yang et al., 2017; Hou et al., 2018; Zou et al., 2019, 2020]. Inoue et al. [2018] have also numerically simulated the eruption of interconnected magnetic flux ropes, which were revealed in the coronal magnetic field of NOAA 12673 reconstructed from a vector magnetogram.

\subsection{Direct superposition of radio images and line-of-sight photospheric magnetogram}

The preliminary stage of NLS identification is the direct superposition of AR microwave images with dis- 
tribution of the line-of-sight component $B_{1}$ of the photospheric magnetic field $\mathbf{B}$. A necessary condition for this procedure is the absence of noticeable radio bursts during observation. Figure 1 shows a superposition of NOAA 12673 radio images and a line-of-sight magnetogram, both obtained on September 6. In the top row is the intensity (Stokes $I$ ); in the bottom row is the circular polarization (Stokes $V$ ). Left five pairs of images were obtained by SRH at five frequencies; the right ones, by NoRH at $17 \mathrm{GHz}$. Similar superpositions for September 5 and 7 do not differ fundamentally from those in Figure 1 and are therefore omitted.

Figure 1 demonstrates that SRH radio maps correspond to each other and to the NoRH one at $17 \mathrm{GHz}$. Brightness centers of the sources in intensity are located in the vicinity of the zero line of $B_{1}$. Based on these facts, this microwave source can be tentatively identified as an NLS.

\subsection{Calculation of the AR coronal magnet- ic field}

The coronal magnetic field above AR is reconstructed in a force-free approximation using the optimization method [Wheatland et al., 2000] as implemented by [Rudenko, Myshyakov, 2009]. As boundary conditions we use SDO/HMI photospheric vector magnetograms [Scherrer et al., 2012] with the $\pi$ ambiguity resolved. During X-class flares occurring on September 6, the AR photospheric magnetic field strength exceeded the maximum value of $5000 \mathrm{G}$, to which SDO/HMI magnetogram data is reliable. Therefore, in some pixels of the magnetograms there is saturation along with other instrumental effects. To reduce these instrumental contributions in the calculations of spatial distribution of the magnetic field, we preliminarily smooth original magnetograms. The calculations are made for a uniform grid consisting of $145 \times 145 \times 71$ knots (length $\times$ width $\times$ height) in steps of $\sim 1 \mathrm{Mm}$.

\subsection{Superposition of radio images and re- constructed distribution of the coronal mag- netic field}

Microwave images of a compact radio source should be compared with the spatial distribution of the magnetic field calculated at a height $h$ above the photosphere. For identifying NLS, the $h$ value can be estimated as the height of the effective brightness center of the source as it approaches the limb. Previous studies on NLSs with NoRH and SSRT suggest that for a typical NLS at $17 \mathrm{GHz} h(17) \approx 2 \mathrm{Mm}$, and for an NLS at $5.7 \mathrm{GHz} h(5.7) \approx 10-12 \mathrm{Mm}$. Note that due to the finite beam sizes of the interferometers there is no need to refine these values. Here, $h=2 \mathrm{Mm}$ is sufficient to eliminate interfering small features in the calculated magnetic field distribution.

For the coronal field distribution $\mathbf{B}$ at $h$, we compute pairs $\left(B_{1}, B_{\mathrm{t}}\right)$ and $\left(B_{\mathrm{r}}, B_{\tau}\right)$. Here $B_{1}$ and $B_{\mathrm{t}}$ are line-of-sight and transversal magnetic components respectively. This pair is convenient to use for analyzing the polarized emission. $B_{\mathrm{r}}$ and $B_{\tau}$ are radial and tangential (horizontal) magnetic components respectively. Being bound to the solar surface, these components are convenient to use for analyzing magnetic structures in AR.

If the times of the radio observation and the reconstructed magnetic field distribution differ, we compensate for solar rotation in radio maps, neglecting the height of the radio source. This does not affect the result, as the values of $h(17)$ and $h(6.8)$ are substantially smaller than the beam sizes of NoRH and SRH respectively.

Figure 2 shows the superposition of $I$ images of NOAA 12673 at $17 \mathrm{GHz}$ (left) and $6.8 \mathrm{GHz}$ (right) with distributions of tangential magnetic component $B_{\tau}$, calculated for 08:36 (hereinafter we refer to the Coordinated Universal Time (UTC)) for $h=2 \mathrm{Mm}$ and $h=10 \mathrm{Mm}$ respectively. The green contours trace the zero lines of the radial magnetic component $B_{\mathrm{r}}=0$ at these heights. In both images, radio brightness maxima are located near the

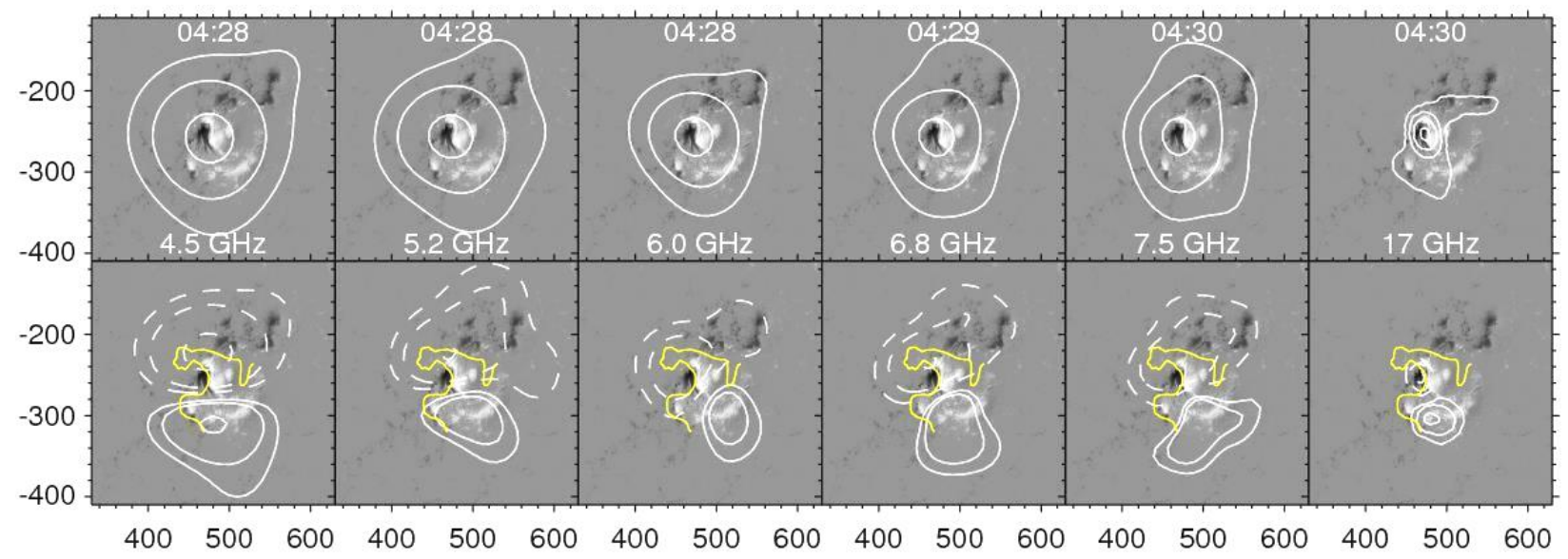

Figure 1. NLS in NOAA 12673 on September 64.5 hours before the first X2.2 flare. Grayscale background represents a photospheric line-of-sight magnetogram (04:30; positive polarity (light), negative polarity (dark)). Contours in the top row show the intensity; levels $[0.1 ; 0.3 ; 0.8]$ of maximum are for SRH; and $[0.03 ; 0.1 ; 0.3 ; 0.8]$, for NoRH. White contours in the bottom row outline the circular polarization (solid: right, dashed: left). Contour levels for SRH are $[0.15 ; 0.3 ; 0.8]$ of the maximum of each polarity; for NoRH, $[0.1 ; 0.3 ; 0.8]$. The yellow contour traces the neutral line. Coordinates are given in arcseconds from the solar disk center 
neutral line and coincide with the region of maximum values of the computed tangential component, thus indicating an NLS. The situation illustrated in Figure 2 also occurred on September 5 and persisted on September 7: the NLS position remained unchanged.

\subsection{Systems of magnetic flux ropes in NOAA 12673}

When computing field lines, we apply the following criteria:

1. Each field line has to be closed on the photosphere, with one of its footpoints being in the vicinity of $3 \mathrm{Mm}$ from a predetermined segment of the main neutral line.

2. The heights of the field lines should not exceed 20 $\mathrm{Mm}$, except for the group of long westernmost field lines whose height is limited to $50 \mathrm{Mm}$.

3 . The magnetic line twist factor $n>1$. It is defined by the expression $n=L|\alpha| /(4 \pi)$, where $L$ is the field line length; $\alpha$ is the force-free parameter in the force-free field equation $\nabla \times \mathbf{B}=\alpha \mathbf{B}$.

Figure 3 illustrates the changes in the configuration of field lines near the neutral line separating opposite-polarity regions of the main magnetic flux: before the X2.2 (onset at 08:57) and X9.3 (11:54) flares on September 6 and before the X1.3 (14:20) flare on September 7 (left images); after these flares (right images). To minimize the overlap of field lines, we present a view from a point located above the center of the base of the computational region.

The field lines matching the criteria specified in this section trace the magnetic flux rope that is in the equilibrium until a certain time, being a reservoir of energy to be released in nonstationary eruptive-flare processes. According to the calculation results, throughout the main neutral line there are several segments of characteristic sheared arcades, i.e. magnetic flux ropes, which constitute an interconnected system. Almost the entire magnetic flux of interconnected arcades has negative helicity. Taking into account the configuration of the radial and transversal components of the photosphere magnetic field, this circumstance is consistent with the electric current equilibrium model in an external magnetic field [van Tend, Kuperus, 1978].

Table lists estimated magnetic flux and total area of photospheric bases of the loop systems with negative helicity, which are shown in Figure 3. When calculating the magnetic flux, we use a magnetic field in the vicinity of $3 \mathrm{Mm}$ from the main neutral line. For each case considered, we have made two estimates: one is related to the field lines rooted to the photosphere in the negative polarity and the other is related to those rooted in the positive polarity. Because a part of the magnetic flux is rooted outside of the given vicinity, there is some difference between the estimates related to the same time. A certain decrease in the magnetic flux occurs after each of the X-class flares, which is manifested in a decreased density of the field lines computed or in the disappearance of some segments of the sheared arcade system. As a result, during each flare AR loses magnetic helicity. The helicity decrease indicates the eruption of a part of the NOAA 12673 electric circuit into the solar wind. Indeed, each of the three X-class flares was eruptive and associated with launch of a CME. The average CME velocities were 391, 433, and $1571 \mathrm{~km} / \mathrm{s}$, according to the CME catalog [https://cdaw.gsfc.nasa.gov/CME_list]. The most powerful X9.3 flare was accompanied by the fastest CME.
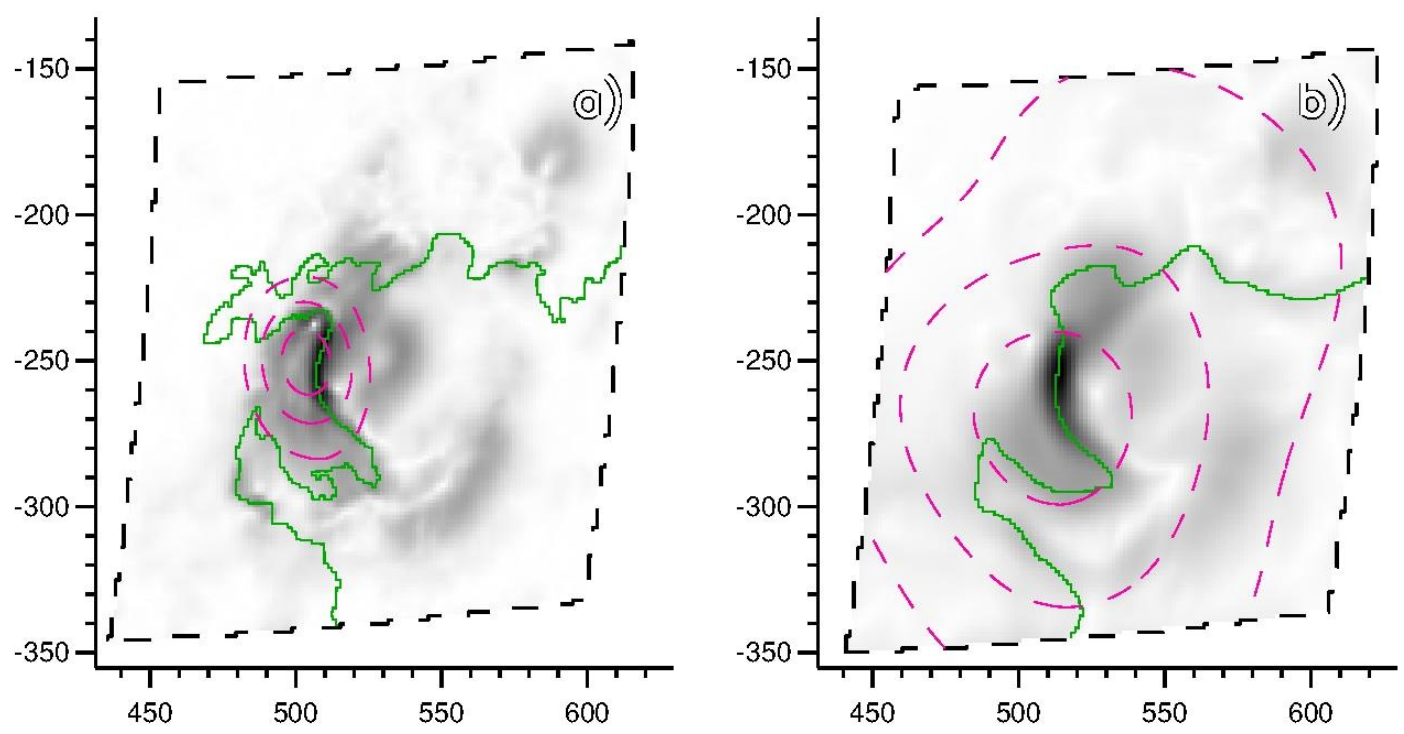

Figure 2. Juxtaposition of a radio source and spatial distribution of the reconstructed magnetic field of AR at a given height on September 6: intensity contours from NoRH data $(17 \mathrm{GHz}, 04: 30), 2 \mathrm{Mm}$ height $(a)$; intensity contours from SRH data (6.8 $\mathrm{GHz}, 04: 29), 10 \mathrm{Mm}$ height $(b)$. Dashed purple contours indicate levels [0.1; 0.3; 0.7] of the maximum radio brightness. All radio maps are converted to the time when the magnetogram was obtained (08:36). Solid green contours show the zero line of the radial magnetic component. Grayscale background is the distribution of the absolute value of the tangential (horizontal) magnetic component. The dashed black line circumscribes the computational region. Coordinates are given in arcseconds from the solar disk center 

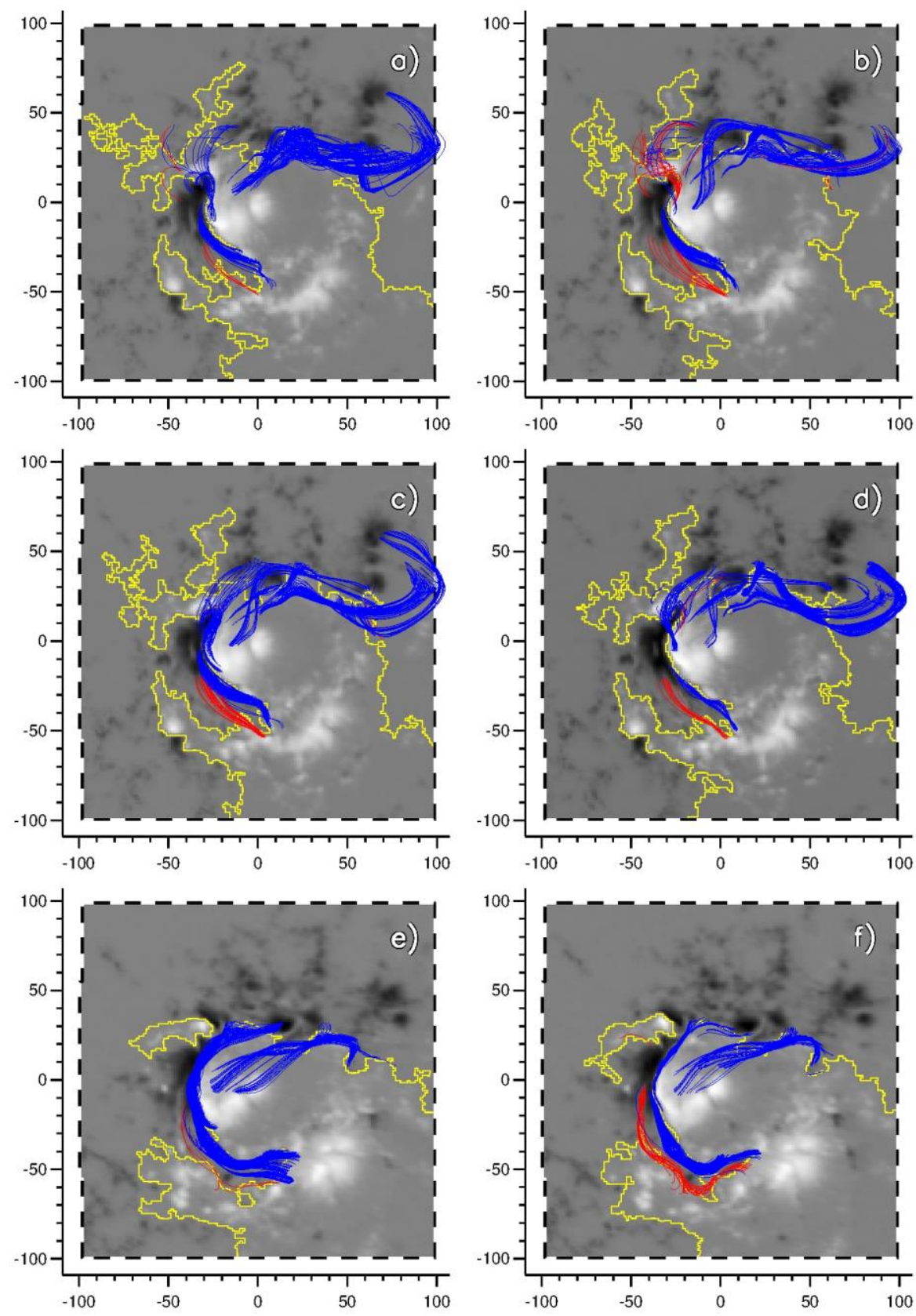

Figure 3. Reconstructed magnetic field lines before (left) and after (right) X-class flares. View from the point above the center of the base of the computational region. Times of the magnetograms used for the calculations: 08:36 (a), 09:48 (b), 11:24 (c), and 12:24 $(d)$ on September 6; 14:00 $(e)$ and 15:36 $(f)$ on September 7. Grayscale background presents the distribution of the radial photospheric field. Yellow contours trace the neutral line. Blue color shows field lines with negative helicity; red, those with positive helicity. The dashed black line circumscribes the computational region. Coordinates are given in arcseconds from the center of the base of the computational region

Estimated magnetic flux constituting the magnetic flux rope with predominant negative helicity. $F$ and $S$ are the magnetic flux and the area it occupies respectively, calculated for field lines outgoing from the regions of positive $(+)$ and negative $(-)$ magnetic polarity

\begin{tabular}{|c|c|c|c|c|c|}
\hline \multicolumn{2}{|c|}{ Date and time } & $F^{+}, 10^{20} \mathrm{Mx}$ & $S^{+}, 10^{17} \mathrm{~cm}^{2}$ & $F, 10^{20} \mathrm{Mx}$ & $S, 10^{17} \mathrm{~cm}^{2}$ \\
\hline \multirow{4}{*}{ September 6 } & $08: 36$ & 2.8 & 4.6 & 3.0 & 3.5 \\
\cline { 2 - 6 } & $09: 48$ & 2.0 & 3.2 & 2.6 & 3.5 \\
\cline { 2 - 6 } & $11: 24$ & 4.3 & 5.4 & 3.3 & 3.1 \\
\cline { 2 - 6 } & $12: 24$ & 2.5 & 4.6 & 1.5 & 2.3 \\
\hline \multirow{2}{*}{ September 7 } & $14: 00$ & 2.5 & 3.2 & 3.9 & 6.9 \\
\cline { 2 - 6 } & $15: 36$ & 0.9 & 3.2 & 2.9 & 6.6 \\
\hline
\end{tabular}




\subsection{Magnetic flux ropes and neutral-line- associated source}

Figure 4 shows a superposition of an I-image at 17 $\mathrm{GHz}$ with the calculated flux-rope magnetic structure of NOAA 12673. Figure 4, $a, b$ illustrates the situation before X2.2 and M7.3 flares. The stable location of the NLS corresponds to the zone of substantially non-potential field structure in combination with a maximum tangential component of the reconstructed magnetic field, thus indicating the presence of a significant electric current in that region. The NLS is projected onto the intersection region of magnetic flux ropes that constitute the extended system arranged along the main polarity inversion line of the AR (see Figure 3).

\section{CONCLUSIONS}

NLS appeared in NOAA 12673 before the multi-day powerful flare activity and persisted all that time. Analysis of radio data from the first stage of SRH and NoRH indicates that after completion of SRH construction its data along with vector magnetograms would be sufficient to identify NLS as a predictor of powerful flares and CMEs.

We have found a structure of interconnected magnetic flux ropes going along the main polarity inversion line from the region of the maximum horizontal magnetic field, where the NLS was located.

Numerical MHD simulation of the flux rope eruption in this region before the X9.3 flare [Inoue et al., 2018] has shown that a single magnetic flux rope is formed due to combining small flux ropes during magnetic reconnection. Such a scenario also provides the basis for the dual-filament CME initiation model [Uralov et al., 2002; Grechnev et al., 2006] developed on the basis of multi-wave observations. In this model, reconnection in the lower corona between two or more segments of a pre-eruptive filament leads to an increase in the length and height of a magnetic flux rope formed from the combined filament. This increases its dipole momentum and total twist, triggering the eruption and reconnection in correspondence with the standard eruptive flare model. These circumstances explain why NLS is the main source of microwave fluctuations in an active region and a precursor of powerful flares.

We thank our colleagues from the ISTP SB RAS Radioastrophysical Department and Radioastrophysical Observatory in Badary, providing SRH observations, and S.A. Anfinogentov and S.V. Lesovoi for their assistance in data processing. We are grateful to the reviewers for constructive suggestions that helped to improve the article. SRH data was obtained using the Unique Research Facility Siberian Solar Radio Telescope [ckprf.ru/usu/73606]. The study was funded by the Russian Science Foundation (project No. 18-12-00172). Methods for joint analysis of data from different instruments and for magnetic field reconstruction were developed with budgetary funding of Basic Research program II.16.

We are indebted to members of the International Consortium for continuing observations with the Nobeyama Radioheliograph until their completion on March 31, 2020, to NASA/SDO teams for the data used in the paper, and to the team who created and maintain the CME catalog generated and maintained at the CDAW Data Center by NASA and The Catholic University of America in cooperation with the Naval Research Laboratory.
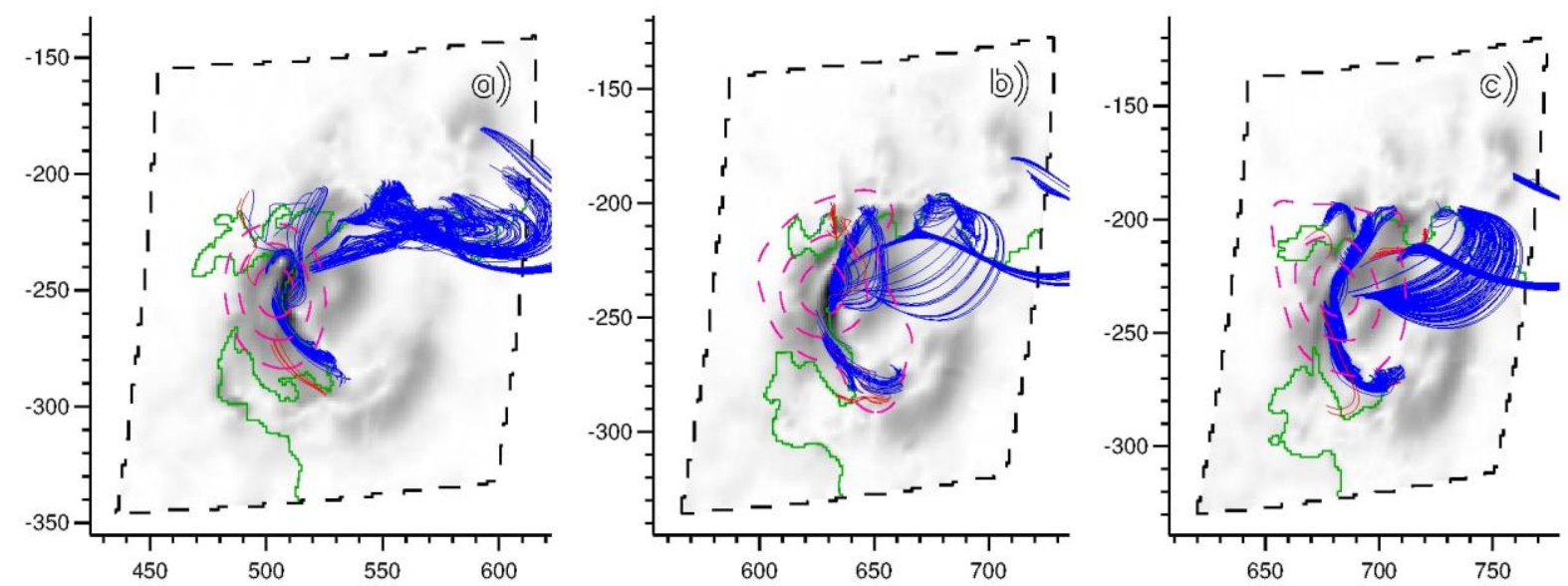

Figure 4. Reconstructed magnetic field distribution at $2 \mathrm{Mm}$ and field lines in comparison with the NLS position at $17 \mathrm{GHz}$ : the 08:36 magnetogram and the 04:30 radio map on September $6(a)$; the magnetogram and radio map at 02:00 on September 7 (b); the 10:00 magnetogram and the 04:32 radio map on September $7(c)$. If necessary, solar rotation is compensated in radio data for the magnetogram time. Dashed purple contours indicate levels $[0.1 ; 0.3 ; 0.7]$ of the maximum NLS brightness. Solid green contours trace the zero line of the radial magnetic field. Grayscale background presents the distribution of the tangential magnetic component by the absolute value. The dashed black line circumscribes the computational region. Blue color shows field lines with negative helicity; red, those with positive helicity. Coordinates are given in arcseconds from the solar disk center 


\section{REFERENCES}

Abramov-Maximov V.E., Borovik V.N., Opeikina L.V., Tlatov A.G. Dynamics of microwave sources associated with the neutral line and the magnetic-field parameters of sunspots as a factor in predicting large flares. Solar Phys. 2015, vol. 290, iss. 1, pp. 53-77. DOI: 10.1007/s11207-014-0605-8.

Abramov-Maximov V.E., Borovik V.N., Opeikina L.V., Tlatov A.G. Evolution of solar active region 12673 by RATAN-600 and SDO data. Trudy XXII Vserossiiskoi ezhegodnoi konferentsii po fizike Solntsa "Solnechnaya i solnechnozemnaya fizika" [Proc. XXII All-Russian Annual Conference on Solar Physics "Solar and Solar-Terrestrial Physics"]. Russia, St. Petersburg, 2018, pp. 15-18. DOI: 10.31725/0552-58292018-15-18. (In Russian).

Akhmedov S.B., Borovik V.N., Gelfreikh G.B., Bogod V.M., Korzhavin A.N., Petrov Z.E., Dikij V.N., Lang K.R., Willson R.F. Structure of a solar active region from RATAN 600 and Very Large Array observations. Astrophys. J. 1986, vol. 301, pp. 460-464.

Altyntsev A.T., Lesovoi S.V., Globa M.V., Gubin A.V., Kochanov A.A., Grechnev V.V., Ivanov E.F., Kobets V.S., Meshalkina N.S., Muratov A.A., Prosovetsky D.V., Myshyakov I.I., Uralov A.M., Fedotova A.Yu. Multiwave Siberian Radioheliograph. Solar-Terr. Phys. 2020, vol. 6, iss. 2, pp. 30 40. DOI: $10.12737 /$ stp-62202003.

Anfinogentov S.A., Stupishin A.G., Mysh'yakov I.I., Fleishman G.D. Record-breaking coronal magnetic field in solar active region 12673. Astrophys. J. Lett. 2019, vol. 880, iss. 2, L29, 5 p. DOI: 10.3847/2041-8213/ab3042

Bakunina I.A., Abramov-Maximov V.E., Osharin A.M. Evolution of the compact microwave inter-sunspot source before strong flare. J. Physics: Conf. Series. 2017, vol. 798, iss. 1, 012041. DOI: 10.1088/1742-6596/798/1/012041.

Bogod V.M., Kaltman T.I., Yasnov L.V. On properties of microwave sources located above the neutral line of radial magnetic field. Astrophys. Bull. 2012, vol. 67, iss. 4, pp. 42 437. DOI: $10.1134 /$ S1990341312040062.

Borovik V.N., Vatrushin S.M., Korzhavin A.N. Radio characteristics of the active region connected with the proton flare of April 24, 1985 based on observations with RATAN600. Bull. Spec. Astrophys. Obs. 1989, vol. 28, pp. 123-131.

Borovik V.N., Abramov-Maximov V.E., Tlatov A.G., Opeikina L.V., Shramko A.D., Yasnov L.V. Signs of preparing powerful solar flares in September 2017 in microwave emission and magnetic field structure of active region from RATAN-600 and SDO/HMI data. Izyestiya Krymskoi astrofiz. obs. [Bull. of the Crimean Astrophys. Observatory]. 2018. Vol. 114, No. 1. P. 89-94. (In Russian).

Chiuderi Drago F., Alissandrakis C.E., Hagyard M. Microwave emission above steady and moving sunspots. Solar Phys. 1987, vol. 112, pp. 89-105. DOI: 10.1007/BF00148490.

Gelfreikh G.B. The problem of search for current sheets in the solar atmosphere with radio astronomical methods. Fizika solnechnykh vspyshek [The physics of the solar flares]. Moscow. Nauka Publ., 1985, pp. 111-117. (In Russian).

Grechnev V.V., Lesovoi S.V., Smolkov G.Ya., Krissinel B.B., Zandanov V.G., Altyntsev A.T., et al. The Siberian Solar Radio Telescope: the current state of the instrument, observations, and data. Solar Phys. 2003, vol. 216, iss. 1-2, pp. 239-272. DOI: 10.1023/A:1026153410061.

Grechnev V.V., Uralov A.M., Zandanov V.G., Baranov N.Y., Shibasaki K. Observations of prominence eruptions with two radioheliographs, SSRT and NoRH. Publ. Astron. Soc. Japan. 2006, vol. 58, pp. 69-84. DOI: 10.1093/pasj/58.1.69.

Hou Y.J., Zhang J., Li T.,Yang S.H., Li X.H. Eruption of a multi-flux-rope system in solar active region 12673 leading to the two largest flares in solar cycle 24. Astron. Astrophys. 2018, vol. 619, A100. DOI: 10.1051/0004-6361/201732530.
Inoue S., Shiota D., Bamba Y., Park S.-H. Magnetohydrodynamic modeling of a solar eruption associated with an X9.3 flare observed in the active region 12673. Astrophys. J. 2018, vol. 867, iss. 1, 83. DOI: 10.3847/1538-4357/aae079.

Kundu M.R., Alissandrakis C.E. Structure and polarization of active region microwave emission. Solar Phys. 1984, vol. 94, iss. 2, pp. 249-283. DOI: 10.1007/BF00151317.

Kundu M.R., Velusamy T. Observation with the VLA of a stationary loop structure on the Sun at 6 centimeter wavelength. Astrophys. J. Lett. 1980, vol. 240, pp. L63L67. DOI: $10.1086 / 183324$.

Kundu M.R., Alissandrakis C.E., Bregman J.D., Hin A.C. 6 centimeter observations of solar active regions with 6 " resolution. Astrophys. J. 1977, vol. 213, pp. 278-295. DOI: 10.1086/155155.

Kundu M.R., Schmahl E.J., Rao A.P. VLA observations of solar active regions at $6 \mathrm{~cm}$ wavelength. Astron. Astrophys. 1981, vol. 94, no. 1, pp. 72-79.

Kuznetsov A.A., Keppens R., Xia C. Synthetic radio views of simulated solar flux ropes. Solar Phys. 2016, vol. 291, iss. 3, pp. 823-845. DOI: 10.1007/s11207-016-0865-6.

Lee J., White S.M., Gopalswamy N., Kundu M.R. Signatures of coronal currents in microwave images. Solar Phys. 1997, vol. 174, iss. 1/2, pp. 175-190. DOI: 10.1023/A:1004956422669.

Lesovoi S.V., Altyntsev A.T., Kochanov A.A., Grechnev V.V., Gubin A.V., Zhdanov D.A., et al. Siberian Radioheliograph: First results. Solar-Terr. Phys. 2017, vol. 3, iss. 1, pp. 3-18. DOI: 10.12737/article_58f96ec60fec52.86165286.

Nakajima H., Nishio M., Enome S., Shibasaki K., Takano T., Hanaoka Y., et al. The Nobeyama radioheliograph. Proc. IEEE. 1994, vol. 82, iss. 5, pp. 705-713.

Quiñones J.A., Korzhavin A.N., Peterova N.G., Santos J. Observations of the solar eclipse on March 7, 1970 with the polarimeter of the Havana Radio Astronomical Station at 4.5 cm. Solnechnye Dannye [Solar Data]. 1975, no. 3, pp. 87-96. (In Russian)

Rudenko I.G., Rudenko G.V., Uralov A.M. Radio source above neutral line in active regions generating long-lasted Xflares. Trudy Vserossiiskoi konferentsii "Mnogovolnovye issledovaniya Solntsa $i$ sovremennye problemy solnechnoi aktivnosti" [Proc. All-Russian Conference "Multiwavelength Investigations of the Sun and Modern Problems of Solar Activity"]. Russia, N. Arkhyz, St. Petersburg, 2007, pp. 462-474. (In Russian).

Rudenko G.V., Myshyakov I.I. Analysis of reconstruction methods for nonlinear force-free fields. Solar Phys. 2009, vol. 257, iss. 2, pp. 287-304. DOI: 10.1007/s11207-009-9389-7.

Scherrer P.H., Schou J., Bush R.I., Kosovichev A.G., Bogart R.S., Hoeksema J.T., Liu Y., Duvall T.L., Zhao J., Title A.M., et al. The Helioseismic and Magnetic Imager (HMI) investigation for the Solar Dynamics Observatory (SDO). Solar Phys. 2012, vol. 275, pp. 207-227. DOI: 10.1007/s11207011-9834-2.

Smolkov G.Ya., Pistolkors A.A., Treskov T.A., Krissinel B.B., Putilov V.A., Potapov N.N. The Siberian Solar Radio Telescope: Parameters and principle of operation, objectives and results of first observations of spatiotemporal properties of development of active regions and flares. Astrophys. Space Sci. 1986, vol. 119, iss. 1, pp. 1-4. DOI: $10.1007 / B F 00648801$.

Strong K.T., Alissandrakis C.E., Kundu M.R. Interpretation of microwave active region structures using SMM soft X-ray observations. Astrophys. J. 1984, vol. 277, pp. 865-873. DOI: 10.1086/161757.

Sych R.A., Uralov A.M., Korzhavin A.N. Radio observations of compact solar sources located between sunspots. Solar Phys. 1993, vol. 144, iss. 1, pp. 59-68. DOI: 10.1007/BF00667982.

Uralov A.M., Sych R.A., Lubyshev B.I., Nefedyev V.P., Golovko A.A., Korobova Z.B., Alissandrakis C.E., Smartt 
R.N., Zhang Hongqi. Microwave source above neutral line as a factor of prediction of powerfull X-ray flares. Issledovaniya po geomagnetizmu, aeronomii i fizike Solntsa [Res. on Geomagnetism, Aeronomy and Solar Physics]. Novosibirsk, Nauka Publ., 1996, iss. 104, pp. 23-48. (In Russian).

Uralov A.M., Sych R.A., Shchepkina V.L., Zubkova G.N., Smolkov G.Ya. Weakly polarized microwave sources in active regions prior to large X-flares. Solar Phys. 1998, vol. 183, iss. 2, pp. 359-368. DOI: 10.1023/A:1005041324807.

Uralov A.M., Nakajima H., Zandanov V.G., Grechnev V.V. Current-sheet-associated radio sources and development of the magnetosphere of an active region revealed from $17 \mathrm{GHz}$ and Yohkoh data. Solar Phys. 2000, vol. 197, iss. 2, pp. 275-312. DOI: 10.1023/A:1026582416968.

Uralov A.M., Lesovoi S.V., Zandanov V.G., Grechnev V.V. Dual-filament initiation of a coronal mass ejection: Observations and model. Solar Phys. 2002, vol. 208, iss. 1, pp. 69-90. DOI: 10.1023/A:1019610614255.

Uralov A.M., Rudenko G.V., Rudenko I.G. Microwave neutral line associated sources: birth, relation to flares, and projection effect. Bull. Russian Academy of Sciences: Physics. 2006a, vol. 70, no. 10, pp. 1688-1691.

Uralov A.M., Rudenko G.V., Rudenko I.G. 17 GHz neutral line associated sources: birth, motion, and projection effect. Publ. Astron. Soc. Japan. 2006b, vol. 58, p. 21. DOI 10.1093/ pasj/58.1.21.

Uralov A.M., Rudenko G.V., Grechnev V.V., Rudenko I.G., Nakajima H., Shibasaki K. Microwave source above neutral line as a source below current sheet. Trudy Vserossiiskoi konferentsi "Mnogovolnovye issledovaniya Solntsa i sovremennye problemy solnechnoi aktivnosti" [Proc. All-Russian Conference "Multiwavelength Investigations of the Sun and Modern Problems of Solar Activity"]. Russia, N. Arkhyz, St. Petersburg, 2007, pp. 484-513. (In Russian).

Uralov A.M., Grechnev V.V., Rudenko G.V., Rudenko I.G., Nakajima H. Microwave neutral line associated source and a current sheet. Solar Phys. 2008, vol. 249, pp. 315-335. DOI: 10.1007/s11207-008-9183-y.

van Tend W., Kuperus M. The development of coronal electric current systems in active regions and their relation to filaments and flares. Solar Phys. 1978, vol. 59, iss. 1, pp. 115-127. DOI: 10.1007/BF00154935.

Vatrushin S.M., Korzhavin A.N. Fizika solnechnoi plazmy [The physics of the solar plasma]. Moscow, Nauka Publ., 1989, p. 100.
Verma M. The origin of two X-class flares in active region NOAA 12673. Shear flows and head-on collision of new and preexisting flux. Astron. Astrophys. 2018, vol. 612, A101, 7 p. DOI: 10.1051/0004-6361/201732214.

Wang H., Yurchyshyn V., Liu C., Ahn K., Toriumi S., Cao W. Strong transverse photosphere magnetic fields and twist in light bridge dividing delta sunspot of active region 12673. Res. Notes American Astron. Soc. 2018, vol. 2, iss. 1, article id. 8. DOI: 10.3847/2515-5172/aaa670.

Wheatland M.S., Sturrock P.A., Roumeliotis G. An optimization approach to reconstructing force-free fields. Astrophys. $J$. 2000, vol. 540, iss. 2, pp. 1150-1155. DOI: 10.1086/309355.

Yang Sh., Zhang J., Zhu X., Song Q. Block-induced complex structures building the flare-productive solar active region 12673. Astrophys. J. Lett. 2017, vol. 849, iss. 2, L21, 7 p. DOI: $10.3847 / 2041-8213 / a a 9476$.

Yasnov L.V. On the nature of neutral-line-associated radio sources. Solar Phys. 2014, vol. 289, pp. 1215-1225. DOI: $10.1007 / \mathrm{s} 11207-013-0396-3$.

Zaitsev V.V. Gyrosynchrotron model of microwave sources associated with the magnetic field neutral line. Geomagnetism and Aeronomy. 2019, vol. 59, pp. 898-903. DOI: 10.1134/ S0016793219070284.

Zou P., Jiang Ch., Feng X., Zuo P., Wang Y. A two-step magnetic reconnection in a confined $\mathrm{X}$-class flare in solar active region 12673. Astrophys. J. 2019, vol. 870, iss. 2, 97, 8 p. DOI: $10.3847 / 1538-4357 / a a f 3 b 7$.

Zou P., Jiang Ch., Fengsi W., Feng X., Zuo P., Wang Y. Continuous null-point magnetic reconnection builds up a torus unstable magnetic flux rope triggering the X9.3 flare in solar AR 12673. Astrophys. J. 2020, vol. 890, iss. 1, 10, 8 p. DOI: 10.3847/1538-4357/ab6aa8.

URL: https://cdaw.gsfc.nasa.gov/CME_list (accessed September 25, 2020).

URL: ckp-rf.ru/usu/73606 (accessed September 25, 2020).

How to cite this article

Kudriavtseva A.V., Myshyakov I.I., Uralov A.M., Grechnev V.V. Microwave indicator of potential geoeffectiveness and magnetic flux-rope structure of a solar active region. Solar-Terrestrial Physics. 2021. Vol. 7. Iss. 1. P. 3-10. DOI: 10.12737/stp-71202101. 